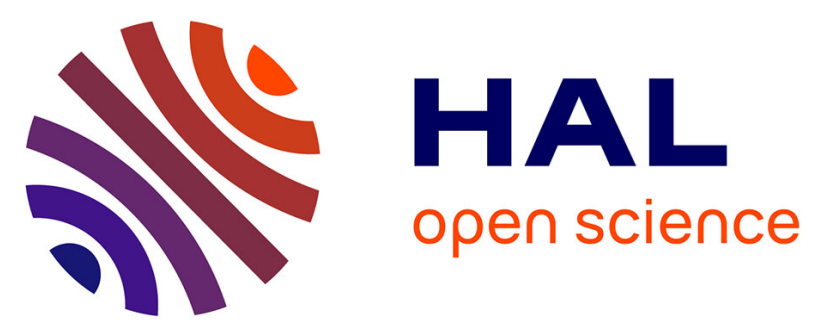

\title{
Energy Conservation for Wireless Mesh Networks: A PSO Approach with Throughput-Energy Consumption Scheme Using Solar Energy
}

Zhe Wang, Taoshen Li, Jin Ye, Zhihui Ge

\section{- To cite this version:}

Zhe Wang, Taoshen Li, Jin Ye, Zhihui Ge. Energy Conservation for Wireless Mesh Networks: A PSO Approach with Throughput-Energy Consumption Scheme Using Solar Energy. 10th International Conference on Intelligent Information Processing (IIP), Oct 2018, Nanning, China. pp.160-169, 10.1007/978-3-030-00828-4_17. hal-02197779

\section{HAL Id: hal-02197779 \\ https://hal.inria.fr/hal-02197779}

Submitted on 30 Jul 2019

HAL is a multi-disciplinary open access archive for the deposit and dissemination of scientific research documents, whether they are published or not. The documents may come from teaching and research institutions in France or abroad, or from public or private research centers.
L'archive ouverte pluridisciplinaire HAL, est destinée au dépôt et à la diffusion de documents scientifiques de niveau recherche, publiés ou non, émanant des établissements d'enseignement et de recherche français ou étrangers, des laboratoires publics ou privés.

\section{(c)(1)}

Distributed under a Creative Commons Attribution| 4.0 International License 


\title{
Energy Conservation for Wireless Mesh Networks: A PSO Approach with Throughput-Energy Consumption Scheme Using Solar Energy
}

\author{
Zhe Wang *, Taoshen $\mathrm{Li}^{* *}$, Jin $\mathrm{Ye}^{* * *}$, Zhihui $\mathrm{Ge}^{* * * * *}$ \\ * College of Electrical Engineering, Guangxi University, Nanning 530004, China \\ designbyyili@163.com \\ ** School of Computer, Electronic and Information, Guangxi University, \\ Nanning 530004, China \\ tshliegxu. edu.cn \\ *** School of Computer, Electronic and Information, Guangxi University, \\ Nanning 530004, China \\ 534262692 @qq. com \\ $* * * *$ School of Computer, Electronic and Information, Guangxi University, \\ Nanning 530004, China \\ gezhihui@foxmail.com
}

\begin{abstract}
A basic problem in the design of Wireless Mesh Networks (WMNs) is presented by the choice of renewable energy for the communication problems in remote regions and post-disaster reconstruction areas. Since the cost and performance are take into consideration as the needs to address the inadequate capacities of links and time-varying traffic demands. In this paper, we aim to find a trade-off between the higher time-varying traffic throughput and lower energy consumption. We propose an optimal approach using particle swarm optimization (PSO) method by formulating this problem into a numerical optimization problem which takes the battery's chargedischarge constraint into consideration firstly. As a further study, an accelerated approximation that computes this optimal problem for larger cases is put forward. Finally, the efficiency of our proposition is proved by numerical results.
\end{abstract}

KEYWORDS: Wireless mesh networks, Energy consumption, Energy efficient, Particle swarm optimization, Numerical optimization 


\section{Introduction}

The research on green networking has become one of the most important research areas as power consumption continues to rise[1-3]. Different methods to reduce the power consumption in different kinds of networks have been put forward in the literature [4-7]. A survey of transmission power control algorithms for cellular networks can be found within [4], they study the convergence property of an iterative power control algorithm and obtained sufficient conditions for convergent. [5] propose an energy-efficient secured routing protocol in MANETs provides security for both link and message. [7] presents a novel routing protocol named Game theory based Energy Efficient Clustering routing protocol (GEEC) which adopted evolutionary game theory mechanism to achieve energy exhaust equilibrium.

Recently, pertinent works on energy efficiency are given in the context of Wireless mesh networks (WMNs) [8-12]. Authors in [9] propose an optimization approach based on an ILP model that minimizes power consumption while ensuring coverage of the active users and enough capacity for guaranteeing quality of service. Another energy optimization study is proposed in [10], where hybrid modulator methods and power plans are take into consideration. [11] proposes a novel configuration for energy management in TDMA-based WMNs. A key distinguishing feature in [12] as an extended work of solution is online flow-based routing approach since existing flows are dynamically consolidated or even re-routed at fixed intervals according to live arrival and departure of mesh clients.

In this paper, the ultimate goal is to economize a lot of energy by optimize the network energy consumption model while the traffic demands must be realized. The first contribution of this work is to provide a deep effective penetration on the modelling of WMNs while the object is to minimize the energy expenditure and the throughput demands are guaranteed as the same time. This simulation contributes to the strategic decision whether a node would be sleep or working in the different time slot during the day. The second program is related to solve the energy-throughput problem and obtain the optimized parameters using PSO algorithm. Then, we propose an APSO which allows us to solve exactly medium size WMNs quickly, and compare the two fast and accelerated approximate methods. At last, we discuss the energy saving performance of our schemes.

\section{System model}

\subsection{Network model}

Consider a WMN with $N$ nodes spread out in a two-dimensional geographical area. We denote the network topology with an undirected graph $G(V, E)$, where $V$ $\left(V=\left\{v_{1}, v_{2}, v_{3}, \ldots, v_{V}\right\},|V|=V\right)$ is the set of nodes and $E$ is the set of wireless connec- 
tion links between nodes. The $V$ has two subset where $V_{G}\left(V_{G}=\left\{g_{1}, g_{2}, \ldots, g_{G}\right\}\right)$ is a sub-class of gateways and $V_{M R}\left(V_{M R}=\left\{m_{1}, m_{2}, \ldots, m_{M}\right\}\right)$ is a sub-class of mesh routers.

Time-slotted system is used in this paper. The considered interval is $[0, \mathrm{~T}]$, and is divided into discrete time samples $t=\left\{t_{0}, t_{1}, t_{2}, \ldots, t_{T}\right\}$, where $t_{k}(k \in\{0,1, \ldots, T\})$ is the index of time slot. Let $C_{i, j}\left(t_{k}\right)(i, j \in\{0,1, \ldots, V\})$ denote the capacity over a wireless link between nodes $\left(v_{i}, v_{j}\right)$ in $t_{k}$, and $S_{i}\left(t_{k}\right)$ be the operating status of node $v_{i}$ in $t_{k}$, then,

$$
\begin{aligned}
& 0 \leq C_{i, j}\left(t_{k}\right) \leq C_{\max } \bullet S_{i}\left(t_{k}\right) \\
& 0 \leq C_{i, j}\left(t_{k}\right) \leq C_{\text {max }} \bullet S_{j}\left(t_{k}\right)
\end{aligned}
$$

Where $C_{\max }$ denote the original capacity of link $\left(v_{i}, v_{j}\right)$, and $S_{i}\left(t_{k}\right)$ is a boolean variable. If $v_{i}$ is active in $t_{k}, S_{i}\left(t_{k}\right)$ is 1 ; otherwise, $S_{i}\left(t_{k}\right)$ is 0 .

\section{$2.2 \quad$ Energy model}

For each node, the input energy is composed of solar energy input as which is one of the common environmental energy sources and is more regular than wind energy, and $\mathrm{AC}$ power that are mainly used for continuing operating and active subcarriers. And the remaining energy is stored in the battery [13]. The continuing operating energy consumption comes from the radiators, AC-to-DC rectifiers, machinery movements, etc. As another factor contributes the energy consumption, the subcarrier energy consumption is determined by the number of active subcarriers and each subcarrier's requirement. Let $P_{i}\left(t_{k}\right)$ denotes the entire energy consumed by node $v_{i}$ in $t_{k}, n_{i}\left(t_{k}\right), e_{s}$ and $e_{0}$ respectively be the number of active subcarriers in $t_{k}$, each subcarrier's energy requirement and continuing operating energy consumption respectively. Thus, the total energy consumption $P_{i}\left(t_{k}\right)$ could be calculated as follows:

$$
P_{i}\left(t_{k}\right)=\left[n_{i}\left(t_{k}\right) \bullet e_{s}+e_{0}\right] \bullet S_{i}\left(t_{k}\right)
$$

As shown in the equation (3), $P_{i}\left(t_{k}\right)$ is determined by $S_{i}\left(t_{k}\right)$ : if the node is active, the constant operating and subcarriers consumption are take into concern, otherwise, there is no energy consumption when the node is inactive. It is deserved that the number of active subcarriers $S_{i}\left(t_{k}\right)$ should be not more than the $N$.

As mentioned before, the input energy of each node is divided into two parts, one comes from the solar energy and transform facility, another is from AC power grid. As we known, the solar energy harvesting abilities is not stable because of the changing external conditions. When the solar energy is insufficient, the AC power would be the dominating input energy. Let $H_{i}\left(k_{t}\right)$ denote the total input energy during $k_{t}$ of node $n_{i}$, that equals the sum of solar energy input and $\mathrm{AC}$ power input.

$$
H_{i}\left(t_{k}\right)=h_{i}^{\text {solar }}\left(t_{k}\right)+h_{i}^{a c}\left(t_{k}\right)
$$


In addition, each node has a battery as an extended device for storing the remainder energy. Let $B_{i}\left(t_{k}\right)$ be the residual energy of node $v_{i}$ 's buttery at the time beginning of $t_{k}$, it is equaled the last time residual energy $B_{i}\left(t_{k-1}\right)$ plus the energy input in $t_{k-1}\left(H_{i}\left(k_{t-1}\right)\right)$ and minus the corresponding energy consumption $\left(P_{i}\left(t_{k-1}\right)\right)$. Also, $B_{i}\left(t_{k}\right)$ should not more than the battery capacity which we can note $B_{i}{ }_{i}$ :

$$
\begin{gathered}
B_{i}\left(t_{k}\right)=B_{i}\left(t_{k-1}\right)+H_{i}\left(t_{k-1}\right)-P_{i}\left(t_{k-1}\right) \\
0 \leq B_{i}\left(t_{k}\right) \leq B_{i}^{0} \\
P_{i}^{\text {dis }}(t) \bullet P_{i}^{\text {chg }}(t)=0 \quad \forall t \in t_{k}, \quad \forall i \in V
\end{gathered}
$$

Eq. (7) is a charge-discharge constraint for the battery as $P_{i}^{d i s}(t)$ denotes the discharge power of the battery and $P_{i}^{\text {chg }}(t)$ denotes the charge power of the battery and $t$ denotes the point in time slot $t_{k}$. This constraint ensures the battery can't discharge or charge in the same time. As the constraint is a complementary problem, relaxation factor $\mathcal{E}$ is produced to solving this problem.

$$
\begin{aligned}
& P_{i}^{d i s}(t) \bullet P_{i}^{\text {chg }}(t) \leq \varepsilon \quad \forall t \in t_{k}, \forall i \in V \\
& \varepsilon \rightarrow 0, \quad P_{i}^{d i s}(t) \geq 0, \quad P_{i}^{\text {chg }}(t) \geq 0
\end{aligned}
$$

\subsection{Traffic model}

Due to the limitation of the available energy and network capacity, the node cannot activate all the subcarriers simultaneously at all times. Assume that the upstream data flow of node $v i$ is $u_{i}\left(t_{k}\right), r^{O}$ note the achievable transmission rate of an active subcarrier. The $u_{i}\left(t_{k}\right)$ should satisfy the following equation:

$$
u_{i}\left(t_{k}\right)=S_{i}\left(t_{k}\right) \bullet n_{i}\left(t_{k}\right) \bullet r^{0}
$$

Thus, the system throughput in $t_{k}$, notes $U\left(t_{k}\right)$, is composed of all the nodes' local data traffic $u_{i}\left(t_{k}\right)$, as the following:

$$
U\left(t_{k}\right)=\sum_{v_{i} \in V} u_{i}\left(t_{k}\right)
$$

For each node, the throughput is qualified for data relaying of other node. Assume that $u_{i, j}\left(t_{k}\right)$ is the actual total traffic via link $\left(v_{i}, v_{j}\right)$, therefore, it should be less than the link capacity:

$$
0 \leq u_{i, j}\left(t_{k}\right) \leq C_{i, j}\left(t_{k}\right)
$$

As another description, we define the node $v_{i}$ is interfered from $v_{j}$ whether $\left\|v_{i}-v_{j}\right\|$ $\leqq R_{i}$ (where $R_{i}$ denote the interference range) and $v_{i}$ is not the intend receiver, which 
extends the physical interference model of [14]. Recall that the $\left\|v_{i}-v_{j}\right\|$ is the Euclidean distance between node $v_{i}$ and $v_{j}$.

Also, the traffic model is subject to the following constrains (12)-(18), where $C_{i}\left(t_{k}\right)$ denote the anticipated uplink data traffic of $v_{j}$ during time slot $t_{k}$. Eq. (13) insures none data relaying over a inexistent link. Eq. (12) is also an mathematical expression of the link capacity constraint.

$$
\begin{gathered}
0 \leq u_{i}\left(t_{k}\right) \leq S_{i}\left(t_{k}\right) \bullet C_{i}\left(t_{k}\right) \\
u_{i, j}\left(t_{k}\right)=0 \quad \forall i, j \in \mathrm{V}_{\mathrm{G}} \\
\sum_{j \in V} u_{i, j}\left(t_{k}\right) \leq u_{i}\left(t_{k}\right) \quad \forall i \in V_{M R} \\
\sum_{j \in V} u_{j, i}\left(t_{k}\right) \leq u_{i}\left(t_{k}\right) \quad \forall i \in V_{M R} \\
\sum_{i \in V_{\text {WR }}} \sum_{j \in V_{G}} u_{i, j}\left(t_{k}\right) \geq 0 \\
\sum_{l \in V_{M P R}}\left[u_{l, j}\left(t_{k}\right)-u_{j, l}\left(t_{k}\right)\right]+\sum_{i \in V_{G}} u_{i, j}\left(t_{k}\right) \mathrm{M}_{i, j}\left(t_{k}\right)=\sum_{i \in V_{G}} u_{i, j}\left(t_{k}\right) \quad \forall j \in V_{M R} \\
\sum_{i, j \in V} \operatorname{sng}\left[u_{i, j}\left(t_{k}\right)\right] \leq H
\end{gathered}
$$

Eq. (13) guarantees the gateway is the last node of a link in the process of traffic routing and has competent capacities to upload all the received traffic. Eq. (14) and (15) eliminate loops while routing a flow. Eq. (16) guarantees the flow could be routed to gateways successfully and eventually. Eq. (17) is a causal information of the flow continuity, where the $M_{i, j}\left(t_{k}\right)$ denotes the traffic load in $t_{k}$. Eq. (18) is a constraint for path length, $H$ is the upper limit of each flow's hops.

\subsection{Optimization problem}

From Eq. (10), each node's data traffic for system in time plot $k_{t}$ has been modeled eventually. Now considering the whole time period $[0, T]$, all the system traffic in each time plot $t_{k}$ make up the system total utility.

$$
u=\sum_{t_{k} \in T} U\left(t_{k}\right)=\sum_{t_{k} \in T} \sum_{v_{i} \in V} u_{i}\left(t_{k}\right)
$$

After the analysis of the previous works, our primary objective is to maximize the system utility $u$ by selecting the parameters $S_{i}\left(t_{k}\right)$ and $n_{i}\left(t_{k}\right)$ legitimately. In another side, considering the energy consumption model since the energy is limited. The total system energy consumption which should be minimized is counted as follows: 


$$
p=\sum_{t_{k} \in T} \sum_{v_{i} \in V} P_{i}\left(t_{k}\right)
$$

In this paper, we present a weighted optimization problem as a combination of the total system utility and the total energy consumption. Since both of the $u$ and $p$ are non-negative and $p$ is positive, the optimization problem can be denoted as (21). We adapt $a$ and $b$ to different weighted coefficient for the total system utility and the total energy consumption. The sampling interval is set to be one hour [15]

$$
\begin{array}{lll}
P: \quad \max \quad & \frac{a \bullet u}{b \bullet p} \\
& \text { sbuject to } & E q .(1)-(17)
\end{array}
$$

\section{Optimization}

\subsection{Using PSO method}

It is obvious that the optimization problem $P$ is non-linear since there are many nonlinear constraints existed (i.e., Eq. (3)). Assumed the traffic load for the system and input solar energy are normally distributed throughout the day with mean 12 and variance 6 . Then the feasible regions of parameters are denoted and problem $P$ is transferred into a precede form. $P$ becomes the following equation:

$$
\begin{aligned}
P 1(\vec{S}, \vec{n}): \max \frac{a}{} \frac{\bullet \sum_{t_{k} \in T} \sum_{v_{i} \in V} u_{i}\left(t_{k}\right)}{b \bullet \sum_{t_{k} \in T} \sum_{v_{i} \in V} P_{i}\left(t_{k}\right)} \\
\text { sbuject to Eq.(1)-(17) }
\end{aligned}
$$

Note that $P l$ cannot be accumulated by each node's traffic divide energy consumption itself because the energy consumption could be zero if the node is not active. Accumulations must be calculated on the molecular and the denominator separately, and $P l$ is finally operated by the division.

Since the PSO is a stochastic procedure, the stop condition has to be defined as the maximal generations are accomplished (or the value of global best fitness and zooid best fitness are unmodified). Finally, the algorithm figures out every node's operating status $S_{i}\left(t_{k}\right)$ and maximal supporting subcarriers $S_{i}\left(t_{k}\right)$ in time $t_{k}$.

\subsection{Using APSO method}

As an essential step of PSO algorithm, the global best and individual best are used during evolution process. The introduce of individual best increases the diversity of the solution. However, this diversity can be simulated or achieved by using some randomness that could accelerate the convergence of the algorithm. A simpli- 
fied version is to use the global best only, we can write the location update process in a single step as

$$
\begin{aligned}
& S_{i}\left(t_{k+1}\right)=S_{i}\left(t_{k}\right)+\beta\left[\text { gbests }-S_{i}\left(t_{k}\right)\right]+\alpha r \\
& n_{i}\left(t_{k+1}\right)=n_{i}\left(t_{k}\right)+\beta\left[\text { gbestn }-n_{i}\left(t_{k}\right)\right]+\alpha r
\end{aligned}
$$

As Eq.(23) shows, the velocity does not appear as there is no need to deal with the velocity vectors initialization. Here the introduction of stochastic term ar makes the system have the ability to escape from any local optimum, while the $a$ is a constant that is related to the size if problem, and $r$ follows the probability distribution. Typically, $\alpha=0.1 L$ to $0.5 L$ where $L$ is the scale of each variable, while $\beta=0.2$ to 0.7 is sufficient for most applications.

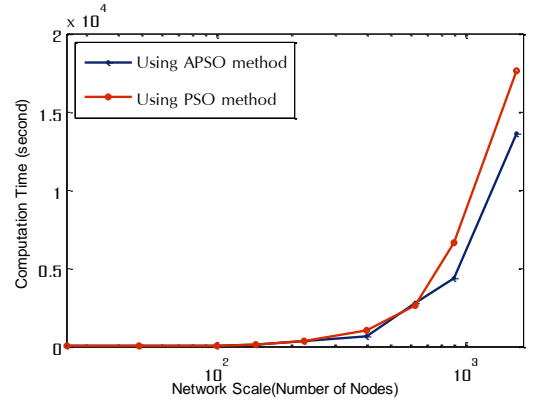

Fig. 1. Calculating times of the two algorithms for growing scale of networks

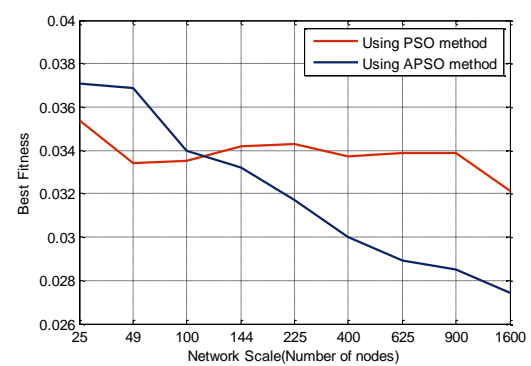

Fig. 3. Best fitness of the two algorithms for growing scale of networks

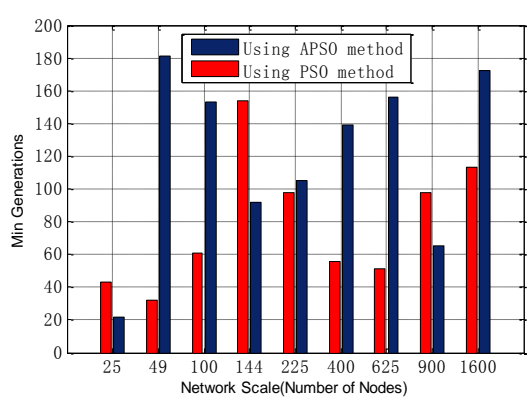

Fig. 2. Minimum generations of the two algorithms for growing scale of network

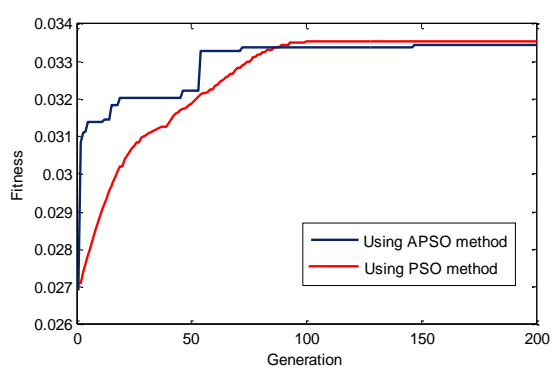

Fig. 4. Fitness modifying using the two algorithms for 144 nodes network

Fig. 2 shows the different calculating time needed for different scales of network

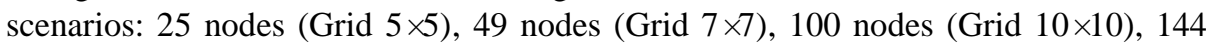

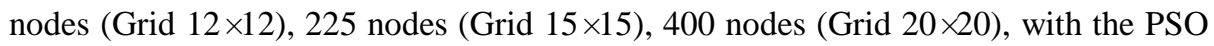
method and APSO method respectively. Each node in scenarios are on a square grid and the gateways are in the four vertexes so that the node density is always the same. Note that both of the two method's max generations equal 200. The computations 
time of the two methods do not have much difference for the small scale networks. With the network scale becomes bigger, the computation time gap between PSO and APSO becomes bigger too.

Fig. 3 depicts the minimum generations of the two algorithms when the value of global best fitness and zooid best fitness are maximum and unmodified. From Fig.3, most network schemes are need more generations for optimize using APSO method than using PSO method. This is because the APSO method only use the global best and the velocity vector is abandoned too. These simplifications make APSO method more stochastic than PSO method.

Fig.4 shows the best fitness form different networks using the two methods respectively. From Fig.5, the best fitness optimized with PSO method is more stable than the APSO method because the APSO method makes the algorithm more randomness. APSO method searches feasible solutions in a wider area that makes the fitness value modifying more precipitous (seeing Fig.5 as an example for 144 nodes network).

\section{Performance evaluation}

In case for testing the system performance, we also demonstrate the expression of Naive formulation which focuses on maximum the total throughput as well as neglects the energy conservation. Parameters are listed in Table 1.

Table 1. Experimental Parameters

\begin{tabular}{c|l}
\hline Parameter & Value \\
\hline$t_{0}$ & 1 hour \\
Operating power $\left(e_{0}\right)$ & $712 \mathrm{~W}$ \\
Number of time slots $(k)$ & 12 \\
Power consumed by per subcarrier $\left(e_{s}\right)$ & $1.06 \mathrm{~W}$ \\
Number of nodes & 20 \\
Number of Gateways & 4 \\
Number of subcarriers $\left(n_{i}\right)$ & $6 \times 100$ \\
Rate per a subcarrier $\left(r^{0}\right)$ & $0.5 \mathrm{Mbps}$ \\
Maximum hop & 8 \\
Battery capacity $\left(B_{i}{ }^{0}\right)$ & $2 \mathrm{~kW}$ \\
\hline
\end{tabular}

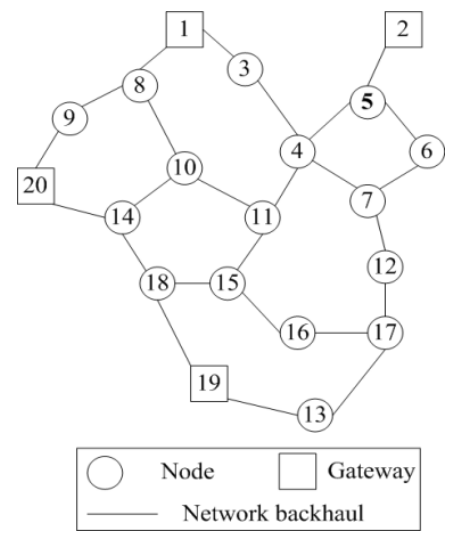

Fig. 5. Network topology used

in performance evaluation

We consider a scheme with 20 nodes, 4 of which are gateways. Each node is connected to its neighbor nodes via a microwave link or long distance Wi-Fi. The bandwidth between two adjacent nodes is randomly selected between 50Mbps and $250 \mathrm{Mbps}$, according to the instructions in product manuals. We set $e_{0}=712 \mathrm{~W}$, $e_{s}=1.06 \mathrm{~W}$ and $n_{i}=600 r_{0}=0.5 \mathrm{Mbps}$ based on the measurement in [15]. The battery capacity $B_{i}{ }^{0}$ is $2 \mathrm{~kW}$, and the initial battery level approaches $100 \%$. We assume the maximum output power of solar energy transform facility in every node is $127 \mathrm{~W}$. The maximum hop sets 8 . Fig.5 shows the network topology in our cases. 


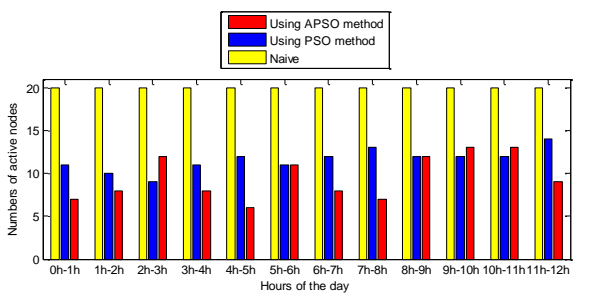

Fig. 6. Numbers of active nodes

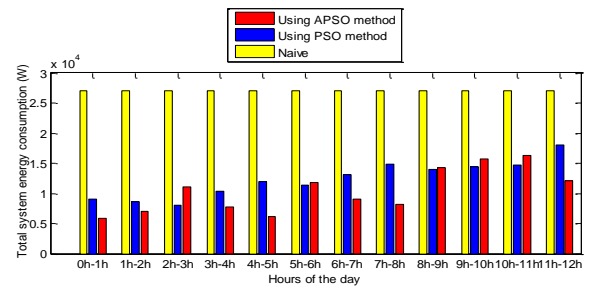

Fig. 8. Total system energy consumption

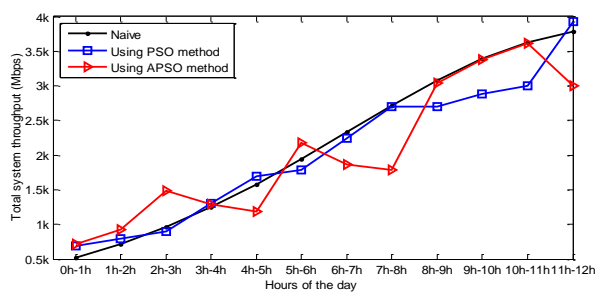

Fig. 7. Total system throughput

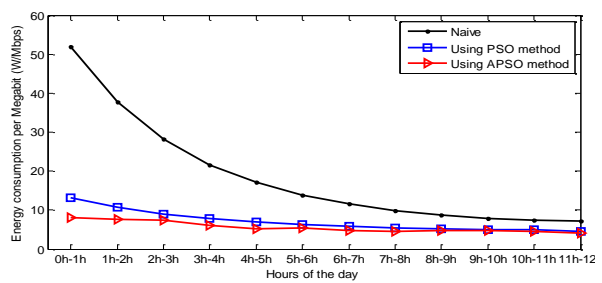

Fig. 9. Energy consumption per megabit

For each node, we suppose the desired data flow is selected from interval [20Mbps, $50 \mathrm{Mbps}$ ] and the gateways have enough capacity to upload all the received traffic.Fig. 6 depicts the number of active nodes at different times of the day with an illustration. Accordingly, during low intervals, some nodes are underutilized. The schemes optimized by PSO and APSO methods have less active nodes than the naive scheme. Due to the uniform distributed traffic demands in geographical position, the numbers of active nodes with PSO method do not have many discrepancies in different hours of the day, and they have a little increase with the growth of the time.

Fig.7 presents the total system throughput with three strategies during a half day. From Fig.8, traffic demands are proportional to the total system throughput. Because of the randomness of the APSO method, there are clear gaps between APSO scheme with excepted information scheme and the PSO one.

Fig. 8 shows the total energy consumption of the three strategies. As a result, we can see that the energy consumption with PSO and APSO scheme are both lower than the naive one, they increase with the traffic grows. Fig. 9 sketches another contrast of energy consumption per megabit. It is apparent that the energy is more efficient with PSO and APSO scheme.

\section{Conclusion}

In this paper, we focus on the energy consumption problem for the WMNs with renewable energy. In order to minimize the energy consumption and guarantee the system throughput, we propose a throughput-energy efficiency model which translates the conundrum into an optimal problem. Then we figure out the optimum solutions that realize the maximal throughput and energy-efficient using PSO method and 
APSO method for large scale networks. Theoretical and simulative results show that the optimization problem can be solved by these methods and lower system energy consumption can be achieved. Providing energy harvesting (EH) capability to network devices will receive more interests to prolong the lifetime on energy limited devices and to realize perpetual operation.

\section{References}

1. Al-Hazmi, Y., De Meer, H., Hummel, K. A., Meyer, H., Meo, M., \& Remondo, D. (2011). Energy-efficient wireless mesh infrastructures.IEEE Network the Magazine of Global Internetworking, 25(2), 32-38.

2. Bianzino, A. P., Chaudet, C., Rossi, D., \& Rougier, J. (2012). A survey of green networking research. IEEE Communications Surveys \& Tutorials, 14(1), 3-20.

3. Wang, X., Vasilakos, A. V., Chen, M., Liu, Y., \& Kwon, T. T. (2012). A survey of green mobile networks: opportunities and challenges. Mobile Networks \& Applications, 17(1), 4-20.

4. Narendran, K., Karthik, R. M., \& Sivalingam, K. M. (2016). Iterative power control based admission control for wireless networks. Wireless Networks, 1-15.

5. Singh, T., Singh, J., \& Sharma, S. (2016). Energy efficient secured routing protocol for manets. Wireless Networks, 1-9.

6. Lin, C. C., Deng, D. J., \& Jhong, S. Y. (2016). Energy-efficient placement and sleep control of relay nodes in heterogeneous small cell networks. Wireless Networks, 1-16.

7. Lin, D., \& Wang, Q. (2016). A game theory based energy efficient clustering routing protocol for wsns. Wireless Networks, 1-11.

8. Boiardi, S., Capone, A., \& Sanso, B. (2012). Energy-aware planning and management of Wireless Mesh Networks. Global Communications Conference (GLOBECOM), 2012 IEEE (pp.3049-3055). IEEE.

9. Lorincz, J., Capone, A., \& Bogarelli, M. (2010). Energy savings in wireless access networks through optimized network management. IEEE International Conference on Wireless Pervasive Computing (pp.449-454). IEEE Press.

10. Luo, J., Rosenberg, C., \& Girard, A. (2010). Engineering wireless mesh networks: joint scheduling, routing, power control, and rate adaptation.IEEE/ACM Transactions on Networking, 18(5), 1387-1400.

11. Amokrane, A., Langar, R., Boutaba, R., \& Pujolle, G. (2012). A green framework for energy efficient management in TDMA-based wireless mesh networks. International Conference on Network and Service Management (pp.322-328). International Federation for Information Processing.

12. Amokrane, A., Langar, R., Boutabayz, R., \& Pujolle, G. (2013). Online flow-based energy efficient management in Wireless Mesh Networks.IEEE Global Communications Conference (pp.329-335).

13. Li, M., Nishiyama, H., Kato, N., \& Owada, Y. (2015). On the energy-efficient of throughput-based scheme using renewable energy for wireless mesh networks in disaster area. IEEE Transactions on Emerging Topics in Computing, 3(3), 420-431.

14. Gupta, P., \& Kumar, P. R. (2000). The capacity of wireless networks.IEEE Transactions on Information Theory, 46(2), 388-404.

15. Mamechaoui, S., Senouci, S. M., Didi, F., \& Pujolle, G. (2015). Energy efficient management for wireless mesh networks with green routers.Mobile Networks \& Applications, 20(5), 1-16. 